\title{
Efficacy and safety of (Sacubitril-Valsartan) Combination on Patients With congestive Heart Failure
}

\author{
M.A.Tabl, H.A.Heneish, K.E.Elrabat, H.A.Khames and H.A.Alkady
}

Cardiovascular medicine, Dept., Faculty of Medicine, Benha Univ., Benha, Egypt

Email: Dr.haitham_heneish@yahoo.com

\begin{abstract}
Background: Heart failure with the preserved expulsion fraction accounts for up to half of the cases of heart failure, is linked to significant morbidity and death. This research aims at assessing the bio-clinical effects of the combination of sakubitril and valsartan in the treatment of congestive patients. Methods: A total of 200 patients in this research were split into two groups: in Group A there were 100 cases started with sacubitril/valsartan; and in Group B there were the remaining 100 cases treated with valsartan alone. All patients have been submitted to comprehensive history, physical examination and standard laboratory testing. In addition, echocardiography and MRI were used to evaluate the left ventricular function. The bio-clinical impact of the sakubitril/valsartan combination after 6 months of therapy is measured via various measures, including NYHA class, inpatient frequency, BNP levels, and leftventricular systolic function. Results: Basal NYHA classes were similar before treatment across the two groups. After therapy, the NYHA class in the combined group improved significantly (group A). Although there was no significant difference in baseline proPNB between the two groups (971,66 and 1021,41 pg/ml respectively in Groups A and B), the marker decreased significantly after treatment (526,71 vs $757,15 \mathrm{pg} / \mathrm{ml}$ in the other group). Conclusion; in patients with heart failure with decreased expulsion fraction, sacubitril/valsartan is linked with a superior recovery profile. It is linked with a substantial increase in EF, a significant drop in proBNP and a reduction in hospitalisation rates.
\end{abstract}

Keywords: Sacubitril, Valsartan, congestive, Heart Failure.

\section{Introduction}

The global epidemic of heart failure (HF) affects at least 26 million individuals globally. This is the number one reason why individuals over 65 have been admitted to hospital. HF health costs are significant and with an ageing population will rise considerably. Although therapy and prevention have progressed considerably, death and morbidity are still high and the quality of life is low. The stated prevalence, incidence, mortality and morbidity rates exhibit regional changes, based on the various etiologies and clinical features of HF patients. [1] HF is a complicated clinical condition that is defined by decreased heart pumping and/or blood filling capabilities. Physiologically, $\mathrm{HF}$ is characterised as insufficient heart output to satisfy metabolic requirements or secondary adequate heart output to compensatory neurohormonal activation (generally manifesting as increased left ventricular filling pressure). Recently, $\mathrm{HF}$ has been divided into three subtypes, namely reduced ejection (HFrEF), preserved expulsion (HFpEF) and medium explosion (HFmrEF) HF, based on ejection fraction, natriuretic peptide levels and the presence of structural heart disease and diastolic dysfunction. HF is divided into three subtypes. [2]

It accounted for an estimated health expense of around $\$ 31$ billion in 2012, equal to more than 10 percent of the United States' total cardiovascular health cost (US). However, projections are much more worrisome, with total expenditures projected to rise by $127 \%$ between 2012 and 2030. [3]

There have been significant advances in the last couple of decades in the treatment of HF using inhibitors of angiotensin-converting enzymes (ACE), aldosterone antagonists, beta-receptor blockers, and resynchronization therapy. Nonetheless, HF still has a dismal prognosis. About half of individuals who acquire HF die within five years of diagnosis. One of the main problems in cardiology is the quest for improved HF therapies. Greater knowledge of molecular dynamics and humoral disturbance leads to a novel therapy for HF. [4]

Recently, a novel combination of Entresto ${ }^{\circledR}$ (sacubitril/valsartan) has been authorised to treat heart failure and decreased ejection in patients. The risk of cardiovascular mortality and hospitalisation in individuals suffering from chronic cardiac failure (NYHA Class II-IV New York Heart Association) and decreased ejection is noted. Entresto may be safely started in a hospital or an outpatient environment and in a broad spectrum of stabilised patients soon after an acute heart failure event. [5]

The medicine provides a new dual action mechanism designed to decrease the stress on the insufficient heart. Valsartan inhibits the negative effects on the cardiovascular system of angiotensin II, while sacubitril prevents neprilysine to improve cardiovascular systems. Biomarkers related to regeneration of the ventricle, myocardial damage and fibrosis may offer mechanical insight and clinical advice on usage of sacubitril/valsartan. [6]

This research aims at assessing the bio-clinical effects of the combination of sakubitril and valsartan in the treatment of congestive patients. In addition, we want to assess the side effects of this combination.

\section{Patients and methods}

This study was be conducted in Wadi El-Neel hospital, Banha university hospital. This study was carried on 200 patients with chronic congestive heart failure. 
The patients were classified into two groups:

- Group I of 100 patients underwent treatment with sacubitril/valsartan combination.

- Group II of 100 patients will underwent treatment with valsartan.

\section{Inclusion Criteria}

- Age more than 18 years old.

- Patients with congestive heart failure class (II-IV) symptoms according to New York Heart Association (NYHA)

- Left Ventricular Ejection Fraction of $40 \%$ or less.

- Plasma B-type natriuretic peptide (BNP) level of at least $150 \mathrm{pg}$ per milliliter (or an N-terminal proBNP [NT-proBNP] level $\geq 600$ pg per milliliter) or, if they had been hospitalized for heart failure within the previous 12 months, a BNP of at least $100 \mathrm{pg}$ per milliliter (or an NT-proBNP $\geq 400 \mathrm{pg}$ per milliliter.

Exclusion Criteria

- Patients with symptomatic hypotension.

- Patients with implanted cardiac devices (e.g., CRT)

- Patients with glomerular filtration rate less than $30 \mathrm{~mL} / \mathrm{min} / 1.73 \mathrm{~m} 2$

- Patients who are non-compliant for ACEinhibitors or ARBs.

All patients underwent the following:

- Full medical history was taken and thorough clinical examination according to pre-designed sheet.

- All patients were followed up for 6 months to study the bio-clinical effects of sacubitril/valsartan combination via numerous parameters:

1. New York Heart Association (NYHA) Class.

2. Frequency of hospitalization by acute exacerbation of $\mathrm{CHF}$

3. B-type natriuretic peptide (BNP) or N-terminal pro-BNP levels were determined at the beginning and at the end of the research

4. Left ventricular systolic function by ECHO and cardiac MRI at the beginning and at the end of the research.

- The side effects of (sacubitril/valsartan) combination were determined by assessment of

1. Renal function (Serum creatinine) was assessed every month till the end of the study.
2. Serum electrolytes (Potassium and Sodium) was performed every month till the end of the study.

Approval was obtained from the local ethics committees in Wadi El-Neel Hospital and Banha university Hospital. Written informed consent was obtained from all patients prior to enrolment.

Statistical analysis

SPSS statistics for windows (Statistical Package for the Social Sciences) version 26 (IBM, Armonk, NY, USA) was used for statistical analysis of the collected data. Shapiro-Wilk test was used to check the normality of the data distribution. All tests were conducted with $95 \%$ confidence interval. P (probability) value $<0.05$ was considered statistically significant. Charts were generated using SPSS' chart builder and Microsoft Excel for windows 2019. Descriptive; Quantitative variables were expressed as mean and standard deviation while categorical variables were expressed as frequency and percentage. Continuous Group differences; Independent sample T and Mann Whitney tests were used for inter-group (between subjects) comparison of parametric and nonparametric continuous data respectively. For pair-wise comparison of data (within subjects), the follow-up values were compared to their corresponding basal value using paired samples $\mathrm{T}$ test, Wilcoxon matched pairs signed ranks test or related-samples Friedman's two-way analysis of variance by ranks with Bonferroni correction of $\mathrm{p}$ value for multiple comparisons. Categorical Group differences; Fisher exact and Chi square tests were used for inter-group comparison of nominal data using the crosstabs function.

\section{Results}

The mean age of the included cases was 51.43 and 52.99 years in Groups A and B respectively, with no significant difference between the two groups. Male patients represented $75 \%$ and $64 \%$ of the included cases in the same groups respectively, while the remaining patients were females. No significant difference was noted between the two groups regarding gender. Table (1)

Basal NYHA class was comparable between the two groups before treatment. After treatment, there was a significant improvement of NYHA class in the combined group (group A). Table (2)

Table (1) Age and gender of the studied groups.

\begin{tabular}{llcccc}
\hline & & $\begin{array}{c}\text { Sacubitril/valsartan group } \\
(\mathbf{n = 1 0 0 )}\end{array}$ & Valsartan group (n= 100) & 95\% CI & p \\
\hline \multirow{2}{*}{ Age (years) } & & $51.43 \pm 6.623$ & $52.99 \pm 8.366$ & $-3.66,0.54$ & 0.145 \\
\multirow{2}{*}{ Gender } & Male & $75.0 \%(75)$ & $64.0 \%(64)$ & $-0.24,0.02$ & 0.091 \\
& Female & $25.0 \%(25)$ & $36.0 \%(36)$ & & \\
\hline
\end{tabular}

Data is expressed as mean and standard deviation or as percentage and frequency. $95 \%$ CI: $95 \%$ confidence interval of the mean difference between both groups. $\mathrm{P}$ is significant when $<0.05$. 
Although there was no significant difference between the two groups regarding basal proPNB (971.66 and $1021.41 \mathrm{pg} / \mathrm{ml}$ in groups $\mathrm{A}$ and $\mathrm{B}$ respectively), Group A showed a significant decrease in that marker after treatment (526.71 vs. $757.15 \mathrm{pg} / \mathrm{ml}$ in the other group). Fig. (1)

Serum creatinine levels were comparable between the two groups at baseline and follow up, apart from five- and six-month readings, which were significantly increased in Group B. Serum creatinine showed a significant decrease when compared to its own baseline value throughout the follow up visits ( $\mathrm{p}<$ 0.05 ) in Group A. fig. (2)

Serum potassium levels showed no significant difference between the two groups on basal and first follow up visits. However, it showed a significant increase in Group B in the following scheduled follow up visits. Like potassium, serum sodium levels expressed the same changes.

At six-month follow up visit, EF, measured by Echo, showed a significant increase in Group A $(40.33 \%$ vs. $31.36 \%$ in the other group). Table (5)

Table (2) NYHA classification before treatment and after 6 months in the studied groups.

\begin{tabular}{|c|c|c|c|c|}
\hline \multicolumn{2}{|c|}{ NYHA classification } & Sacubitril/valsartan group $(n=100)$ & Valsartan group $(n=100)$ & $\mathbf{p}$ \\
\hline \multirow{3}{*}{ Basal } & 2 & $27.0 \%(27)$ & $23.0 \%(23)$ & \multirow{3}{*}{0.738} \\
\hline & 3 & $68.0 \%(68)$ & $73.0 \%(73)$ & \\
\hline & 4 & $5.0 \%(5)$ & $4.0 \%(4)$ & \\
\hline \multirow{3}{*}{ Six months } & 1 & $61.9 \%(60)$ & $37.5 \%(36)$ & \multirow{3}{*}{$<0.001$} \\
\hline & 2 & $38.1 \%(37)$ & $41.7 \%(40)$ & \\
\hline & 3 & $0.0 \%(0)$ & $20.8 \%(20)$ & \\
\hline
\end{tabular}

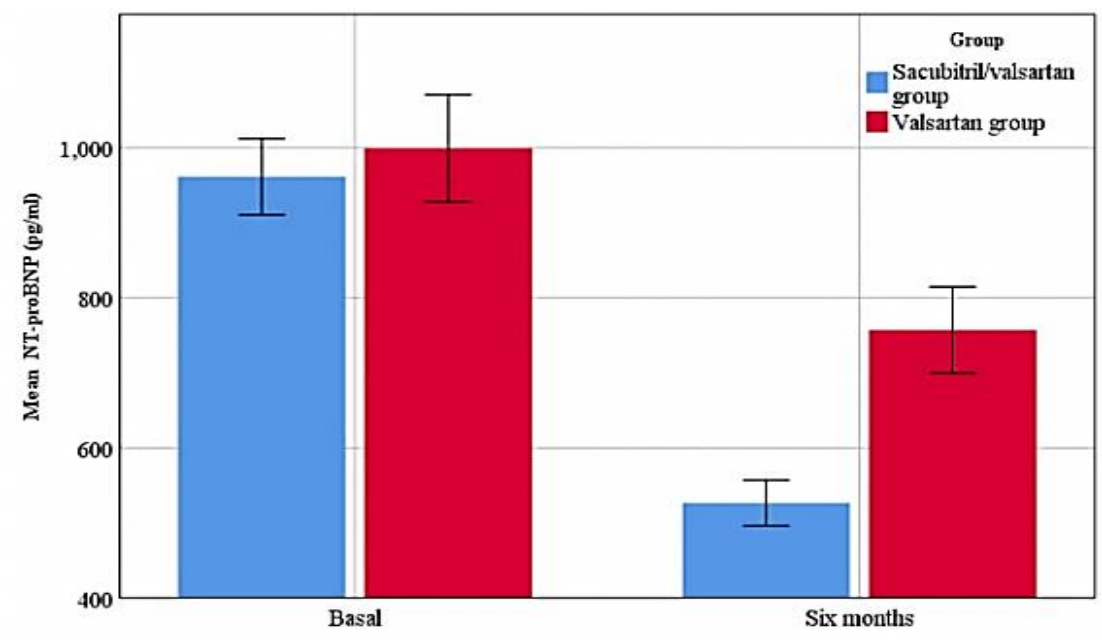

Fig. (1) NT-proBNP (pg/ml) before treatment and after 6 months in the studied groups.

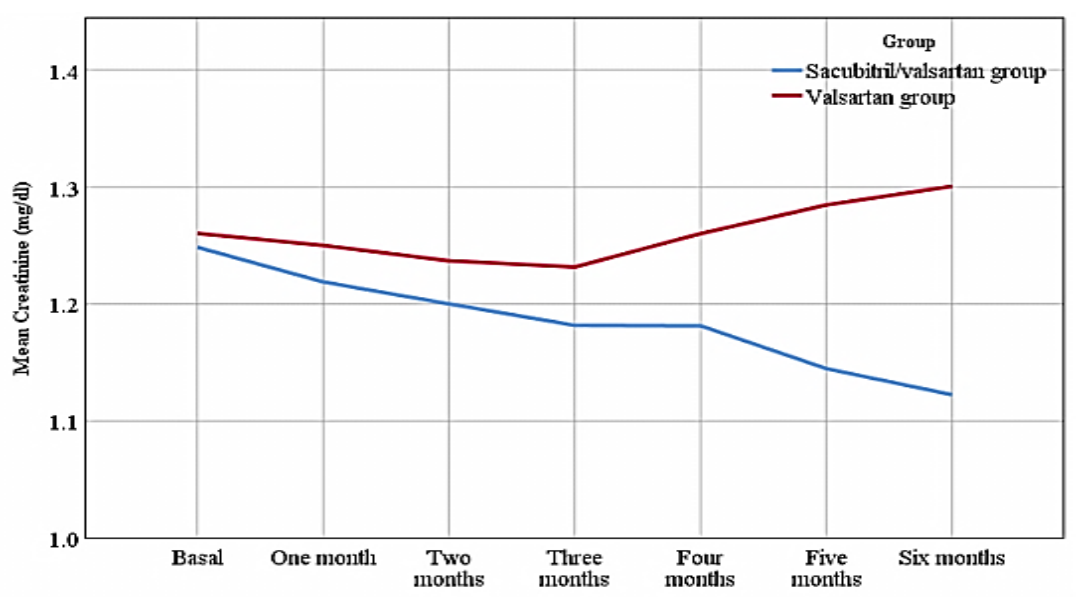

Fig. (2) Basal and follow-up values of serum creatinine in the studied groups. 
Table (3) Comparison of EF by ECHO before treatment and after 6 months in the studied groups.

\begin{tabular}{lcccc}
\hline EF by ECHO & Basal & Six months & 95\% CI & p \\
\hline Sacubitril/valsartan group & $31.68 \pm 4.799$ & $40.33 \pm 4.824$ & $-8.84,-8.19$ & $<\mathbf{0 . 0 0 1}$ \\
Valsartan group & $31.19 \pm 5.417$ & $31.36 \pm 5.864$ & $-8.76,-8.13$ & $<\mathbf{0 . 0 0 1}$ \\
\hline
\end{tabular}

Data is expressed as mean and standard deviation. 95\% CI: 95\% confidence interval of the mean difference between basal and six months values. $\mathrm{P}$ is significant when $<0.05$.

Table (4) Comparison of EF by MRI before treatment and after 6 months in the studied groups.

\begin{tabular}{lcccc}
\hline EF by MRI & Basal & Six months & 95\% CI & p \\
\hline Sacubitril/valsartan group & $31.82 \pm 4.852$ & $40.41 \pm 4.803$ & $-8.76,-8.14$ & $<\mathbf{0 . 0 0 1}$ \\
Valsartan group & $31.35 \pm 5.681$ & $31.54 \pm 5.843$ & $-0.349,0.224$ & 0.666 \\
\hline
\end{tabular}

Data is expressed as mean and standard deviation. 95\% CI: 95\% confidence interval of the mean difference between basal and six months values. $\mathrm{P}$ is significant when $<0.05$.

Table (5) Frequency of hospitalization and incidence of 6-month mortality of the studied groups.

\begin{tabular}{llccc}
\hline & & Sacubitril/valsartan group (n= 100) & Valsartan group (n= 100) & p \\
\hline & $\mathbf{0}$ & $64.0 \%(64)$ & $45.0 \%(45)$ & \\
Frequency of hospitalization & $\mathbf{1}$ & $33.0 \%(33)$ & $28.0 \%(28)$ & $<0.001$ \\
& $\mathbf{2}$ & $2.0 \%(2)$ & $21.0 \%(21)$ & \\
Mortality & $\mathbf{3}$ & $1.0 \%(1)$ & $6.0 \%(6)$ & 0.683 \\
\hline
\end{tabular}

Data is expressed as percentage and frequency. $\mathrm{P}$ is significant when $<0.05$.

\section{Discussion}

While there was a noticeable difference between the two basal PNB groups (971.66 and $1021.41 \mathrm{pg} / \mathrm{ml}$ in Groups A and B, respectively $-\mathrm{p}=0,269)$, the marker decreased significantly (526.71 vs 757.15 $\mathrm{pg} / \mathrm{ml}$, other group < 0.001). In the study of intragroups, it can be shown that, compared to its base value, both groups exhibited a substantial reduction of proPNB following treatment.

Similarly, Gao and his colleagues found that average proPNBs of 10356.94 and 9518.17 were presented in combination and individual therapy groups with no significant difference between them $(p=0.670)$. After therapy, the average values for the identical groups were 3881.59 and 6278.35. The combined regime in this biomarker was linked with a further reduction [7].

Solomon and his colleagues found that NTproBNP changes from the baseline to 12 weeks in a combined group differed substantially from the valsartan group $(p=0 \bullet 005$ with a higher decrease of the combination-treated patients). In the combined group, the value dropped from 783 to $605 \mathrm{pg} / \mathrm{ml}$, whereas for the valsartan group the values were 862 and $835 \mathrm{pg} / \mathrm{ml}$ [8]. This impact was subdued until the reading of 36 weeks.

The same marker also revealed a substantial reduction in comparison to $\mathrm{ARB}$ alone with the same combination. BNP had mean levels of 175.6 and 219.7 $\mathrm{ng} / \mathrm{ms}$ in six months, while the combination and irbesartan groups had mean values of 210.2 and 247.5 $\mathrm{ng} / \mathrm{ml}$ in 12 months $(\mathrm{p}<0.001)$ [9].

In the PIONEER-HF and PARADIGM-HF tests, the same combination also showed that plasma NT-
proBNP was reduced compared to enalapril[10]. Our results on the impact of the medication combination on this marker are supported by all prior research.

The significant decrease in this biomarker naturally has its clinical consequences. In patients with cardiovascular disorders, Plasma BNP and NTproBNP have forecast values, and the decrease of BNP and NT-proBNP predicts an improvement of clinical symptoms [11].

Plasma BNP and NT-proBNP are also utilised therapeutically in the treatment of $\mathrm{HF}$ and cardiac dysfunction patients and are used as forecasts which may assist physicians in adjusting therapy strategies and determining efficacy of therapy to increase survival of the patient[12].

In this research, an echo-measured ejection fraction showed mean values in Groups A and B of 31,68 and 31,19 percent, respectively, without a significant difference between the two groups ( $\mathrm{p}=$ $0,499)$. At a follow-up visit in six months the figures indicated a substantial rise in Group A $(40.33 \%$ vs $31.36 \%$ in the other group $-\mathrm{p}<0.001$ ).

Gao et al. have shown substantial improvement in EF in both groups ( $p<0.001)$, according to our results. For the combined group, EF had mean values of 31.12 and 45.35 percent, with a mean of 30.41 and 36.47 percent for each individual group, before and after the therapy. Although ACE inhibitors and ARBs have been linked with symptom improvements, improved functionality and a decrease in hospital stay in these patients, the current recommendations indicate that no therapy has been proven to effectively reduce morboids or mortality [13]. 
The incidence of hospitalisation in the present research revealed a substantial reduction in Group A $(\mathrm{p}<0.001)$.

In accordance with our results, several authors indicated the ability of this first-class inhibitor of angiotensin receptor neprilysin (ARNI) to decrease hospitalisation in EF patients below the usual range of conditions [8]. The earlier results were verified by other authors [7].

Desai and his colleagues found that the risk of main composites (hazard ratio [HR], 0.80; confidence interval 95 per cent [CI], 0.73-0.87]) and each of its components, i.e., HF (HR, 0.79; 95 per cent CI, 0.710.89) was decreased significantly[14].

In patients with heart failure with intact ejection fractions other authors did not discover a significant advantage to sacubitril-valsartan in relation to the key composite result of total hospitalisation for heart failure [15].

Our results revealed that there was no significant difference between the two groups in 2 and 4 instances in Groups A and B correspondingly ( $\mathrm{p}=0.682)$.

Another research showed that in $1 \%$ of patients in both study groups, death was found with no significant difference in statistical analysis [8].

Other investigators, on the other hand, found that in decreased EF patients this first-class inhibitor of angiotensin receptor neprillysine (ARNI) was effective to prevent cardiovascular death [15].

\section{Conclusion}

On the basis of prior results, it is obvious that sacubitril/valsartan is linked with patients with heart failure with a decreased expulsion rate with a superior recovery profile. It is linked with a substantial increase in $\mathrm{EF}$, a significant drop in proBNP and a reduction in hospitalisation rates.

\section{References}

[1] R. Coronel, J. R. De Groot, and J. J. Van Lieshout, "Defining heart failure." Elsevier Science, vol. 1, pp. 54-67, 2001.

[2] P. Ponikowski et al., "ESC Scientific Document Group. 2016 ESC Guidelines for the diagnosis and treatment of acute and chronic heart failure: The Task Force for the diagnosis and treatment of acute and chronic heart failure of the European Society of Cardiology (ESC) Developed with the special contribution of the Heart Failure Association (HFA) of the ESC," Eur Hear. J, vol. 37, , pp. 2129-2200, 2016.

[3] D. Mozaffarian et al., "Executive summary: heart disease and stroke statistics-2016 update: a report from the American Heart Association," Circulation, vol. 133, pp. 447-454, 2016.

[4] T. Shah, N. Palaskas, and B. Bozkurt, "Heart Failure, Introduction," in Pathophysiology and Pharmacotherapy of Cardiovascular Disease, Springer, vol. 2015, pp. 3-20, 2015.
[5] R. Wachter et al., "P886 Initiation of sacubitril/valsartan in hospitalized patients with heart failure with reduced ejection fraction after hemodynamic stabilization: primary results of the TRANSITION study," Eur. Heart J., vol. 39, pp. ehy564-P886, 2018.

[6] J. L. Januzzi et al., "Rationale and methods of the prospective study of biomarkers, symptom improvement, and ventricular remodeling during sacubitril/valsartan therapy for heart failure (PROVE-HF)," Am. Heart J., vol. 199, pp. 130 136, 2018.

[7] Y. Gao et al., "The impact of Sacrubitril/valsartan on clinical treatment and hs-cTnT and NTProBNP serum levels and the left ventricular function in patients with chronic heart failure," Int. Heart J., vol. 23, pp. 19-231, 2020.

[8] S. D. Solomon. "The angiotensin receptor neprilysin inhibitor LCZ696 in heart failure with preserved ejection fraction: a phase 2 double-blind randomised controlled trial," Lancet, vol. 380, pp. 1387-1395, 2012.

[9] R. Haynes ., "Effects of sacubitril/valsartan versus irbesartan in patients with chronic kidney disease: a randomized double-blind trial," Circulation, vol. 138, pp. 1505-1514, 2018.

[10]P. M. Seferovic . "Clinical practice update on heart failure 2019: pharmacotherapy, procedures, devices and patient management. An expert consensus meeting report of the Heart Failure Association of the European Society of Cardiology," Eur. J. Heart Fail., vol. 21, pp. 1169-1186, 2019.

[11]S. S. Khanam ."Prognostic value of short-term follow-up BNP in hospitalized patients with heart failure," BMC Cardiovasc. Disord., vol. 17, pp. 110, 2017.

[12]R. M. G. Rodriguez . "Homocysteine predicts increased NT-pro-BNP through impaired fatty acid oxidation,” Int. J. Cardiol., vol. 167, pp. 768775, 2013.

[13]A. F. Members ."ESC Guidelines for the diagnosis and treatment of acute and chronic heart failure 2012: The Task Force for the Diagnosis and Treatment of Acute and Chronic Heart Failure 2012 of the European Society of Cardiology. Developed in collaboration with the Heart Failure Association (HFA) of the ESC," Eur. Heart J., vol. 33, pp. 1787-1847, 2012.

[14] A. S. Desai."Effect of the angiotensin-receptorneprilysin inhibitor LCZ696 compared with enalapril on mode of death in heart failure patients," Eur. Heart J., vol. 36, pp. 1990-1997, 2015.

[15] S. D. Solomon . "Angiotensin-neprilysin inhibition in heart failure with preserved ejection fraction," N. Engl. J. Med., vol. 381, pp. 1609 1620, 2019. 